http://dx.doi.org/10.18778/1505-9065.11.17

Paul Bernard-Nouraud

École des Hautes Études en Sciences Sociales de Paris

\title{
UN PRÉSENT EMPREINT DE PASSÉ. BRÈVE GÉNÉALOGIE DE L'HYPOTYPOSE COMME FIGURE DE LA MÉMOIRE
}

\begin{abstract}
"A Present Imprinted with Past. A Brief Genealogy of Hypotyposis as a Memory Figure"
SUMMARY - Through a brief genealogy of the hypotyposis, this essay offers a theoretical description of its different contemporary purposes in relation to memory. We indeed consider that it nowadays constitutes a central memorial figure in contemporary literature and art, which, by evoking the past as if it were present (Dumarsais), disturbs reception. Ultimately, it evidences the difficulty we are now facing to unlock an analogy between the real and its depiction, whether it is poetic or rhetorical, to such a point that it jeopardizes a large section of philosophical thinking.
\end{abstract}

KEYWORDS - hypotyposis, memory, testimony

RÉSUMÉ - À partir d'une brève généalogie de la figure de l'hypotypose, cet article propose une réflexion théorique sur ses usages contemporains dans sa relation à la mémoire. On considère en effet qu'elle constitue aujourd'hui une figure mémorielle centrale de la littérature, voire de l'art contemporains qui, en évoquant le passé comme s'il était présent (Dumarsais), trouble la réception. En toute fin de compte, elle met en évidence la difficulté à dégager une analogie entre le réel et sa description, qu'elle soit poétique et rhétorique, au point de compromettre avec elle tout un pan de la pensée philosophique.

MoTS-CLÉS - hypotypose, mémoire, témoignage

L'hypotypose est une figure à ce point ancienne et répandue, tellement ancrée dans la tradition rhétorique et débordant de ce fait sur la poétique, qu'elle paraît presque consubstantielle à la littérature. Prendre toutefois conscience de son ancienneté n'implique évidemment pas qu'elle soit demeurée jusqu'à présent inchangée, que l'on en use aujourd'hui de la même façon ni avec les mêmes desseins que dans l'Antiquité ou qu'à la période classique. Ses usages actuels indiquent même au contraire que sa fonction, et, en toute fin de compte, son sens, se sont profondément altérés. Pourtant, on découvrira sans peine dans ses effets (et jusqu'en son étymologie) que la dimension spécifiquement mémorielle qu'elle a prise dans la littérature contemporaine (voire dans l'art contemporain en général) se trouvait déjà à l'état latent dans les différentes formes qui avaient été les siennes auparavant, au point que c'est en elles que l'on peut déceler l'énergie mémorielle qui motive l'hypotypose et qui a, en toute fin de compte, pris le dessus sur sa fonction proprement rhétoricienne.

Pour le dire autrement, et afin de préciser les contours de l'enjeu qui oriente cet article, il faudrait d'abord s'étonner avec Roland Barthes de ce que «le 
monde est incroyablement plein d'ancienne Rhétorique $»^{1}$, et apercevoir en conséquence avec lui combien ce qui en reste diffère d'elle et y retourne cependant. L'hypotypose est, à cet égard, une figure symptomatique de ce double mouvement de va-et-vient, et plus que cela. C'est elle (qu'on la nomme ekphrasis, euidentia ou même descriptio, on redéfinira les limites de ces termes par la suite) qui introduit dans l'art du discours ce goût du morceau, de la pièce détachée, si l'on ose dire, et qui participe de sa fonction ostentatoire en emportant finalement la rhétorique tout entière dans son sillage. Celle-ci est alors « entièrement déportée vers l'ornement, écrit Barthes, c'est-à-dire vers ce qui est réputé inessentiel $»^{2}$, déportement dont le point culminant, le moment où l'orateur emporte véritablement son auditoire et le tient sous sa coupe, constitue précisément l'hypotypose, alors même que ce qu'elle décrit peut n'être pas réel mais relever du pur fantasme.

Or, outre la passion classificatrice qui a quelque peu étouffé la valeur philosophique et critique de la rhétorique (mais qui est une passion dont le début au moins peut être historiquement borné), cette apparente gratuité des images qu'elle suscite a d'emblée et jusqu'à aujourd'hui fait craindre «que l'art de 'bien dire' s'affranchisse du souci de 'dire vrai' $\rangle^{3}$, ainsi que le faisait remarquer Paul Ricœur à propos d'une autre figure cette fois, la métaphore, dont, à rebours de cette tendance, il cherchait à rappeler la " vivacité » herméneutique, recherche que l'on se propose de poursuivre ici à partir de l'hypotypose. La thèse de Ricœur se fonde en effet explicitement sur le postulat aristotélicien selon lequel «bien métaphoriser, c'est apercevoir le semblable ${ }^{4}$, et envisage ce principe analogique du "voir comme » en tant que détour révélant un " être comme », la fiction, qu'elle soit poétique ou proprement rhétorique, permettant de décrire des niveaux de réel indescriptibles hors cette voie métaphorique, précisément.

Une telle approche, qui vise à réhabiliter la rhétorique en réévaluant la teneur référentielle de l'une de ses figures majeures, comporte cependant un angle mort : comment décrire un réel tel qu'il enraye le principe métaphorique jusqu'à faire apparaître la figure qui chercherait malgré tout à en rendre compte littéralement déplacée ? Que reste-t-il alors d'une figure qui apparaît radicalement incommensurable au réel qu'elle désigne? Une telle incommensurabilité ne jette-t-elle pas le trouble sur le procès analogique lui-même dont elle tire et sa raison d'être et sa puissance ostensive? Autant de questions qui ne sont aucunement spéculatives, mais que les tentatives de rendre compte des expériences de violence extrême du $\mathrm{XX}^{\mathrm{e}}$ siècle ont au contraire directement posées, et celles écrites d'après Auschwitz au premier chef. Non seulement, comme l'a relevé Philippe Mesnard sur ce sujet,

\footnotetext{
${ }^{1}$ R. Barthes, L'Aventure sémiologique [1985], Paris, Seuil, 1991, «L'ancienne rhétorique » [1965], p. 85.

${ }^{2}$ Ibid., p. 107.

${ }^{3}$ P. Ricœur, La Métaphore vive [1975], Paris, Seuil, 1997, p. 15.

${ }^{4}$ Dans la traduction de Janet Hardy: «car bien faire les métaphores [metaphorein] c'est bien apercevoir [theorein] les ressemblances [to homoion] ». Aristote, Poétique, trad. du grec ancien par J. Hardy, Paris, Gallimard, 2005, 22, 1459a, p. 125.
} 
« une métaphore n'est pas indemne de la réalité qu'elle désigne quand l'humanité est mise en jeu dans cette réalité $»^{5}$, mais, dans une certaine mesure (et c'est bien là ce qu'il s'agit de mesurer à travers l'hypotypose contemporaine), tout le principe analogique qui la fonde s'en trouve compromis, depuis Aristote jusqu'à Kant, et avec lui une esthétique du discernement qui reposait en première et dernière instance sur la copule semblable-vraisemblable.

Il n'est pas lieu d'écrire ici l'histoire de cette rupture dans l'ordre de la pensée, seulement de pointer la capacité de résistance de l'hypotypose dans un tel contexte, elle qui admet traditionnellement qu'il puisse y avoir de l'invraisemblable et du dissemblable, s'écartant ainsi du champ des analogies, en ce qu'elle est une figure qui n'opère pas par déplacement de sens mais davantage par déplacement de temps, ou qui au moins peut en suspendre le cours en se fichant dans sa masse, pour paraphraser Henri Morier ${ }^{6}$. On doit également renoncer (au moins temporairement) à inscrire cette brève généalogie des pouvoirs de l'hypotypose dans l'immense problématique de l'ergon et du parergon quoiqu'elle y ait toute sa place. On se contentera de pointer le fait qu'elle est à la fois œuvre et acte, opus et modus (soit les deux sens d'ergon chez Aristote, on pourrait même dire « œuvre active »), et ornement ou supplément (parergon) à l'œuvre véritable.

Sa fortune critique actuelle vient alors peut-être aussi de cette double qualité qui brouille les limites entre ce qui est réputé être l'objet véritable de l'esthétique - l'œuvre pure - et ses à-côtés. "Où commence et où finit un parergon ", s'interroge ainsi Jacques Derrida dans sa lecture de la Critique de la faculté de juger où Kant exclut les parerga de l'appréciation de la beauté authentique ${ }^{8}$. Il faut pourtant bien en user afin de faire voir les analogies, et c'est tout le sens du $\S 59$ de la troisième Critique que de solliciter un tel cadre en mettant en œuvre des parerga, en l'occurrence les hypotyposes schématique et symbolique, afin d'amorcer le procès analogique qui mène aux Idées, et ce cadre n'est fécond que parce que l'imagination dispose d'images à même de faire voir les ressemblances. Sans elles, sans l'hypothèse d'une certaine adéquation (qui elle-même suppose

\footnotetext{
${ }^{5} \mathrm{Ph}$. Mesnard, Témoignage en résistance, Paris, Stock, 2007, p. 159.

${ }^{6}$ Qui définit l'hypotypose comme une pique dans la «masse » du récit, différente cependant de la digression en ce qu'elle y participe et « contribue à l'actualiser et fait toucher du doigt la réalité », jusqu'à « fixer dans la mémoire un moment capital de la narration ». H. Morier, Dictionnaire de poétique et de rhétorique [1961], Paris, Presses Universitaires de France, 1989, « Hypotypose », p. 525 et 530 .

${ }^{7}$ J. Derrida, La Vérité en peinture [1978], Paris, Flammarion, 1996, « Parergon », p. 66. Sur cette problématique de l'ornement dans le domaine de la stylistique, $c f$. notamment G. Molinié, «Problématique de la répétition ", Langue française, $\mathrm{n}^{0}$ 101, 1994, p. 102-111, en particulier p. 102-103.

${ }^{8}$ E. Kant, Critique de la faculté de juger [1790], trad. de l'allemand par A. Renaut, Paris, GarnierFlammarion, 1995, Première partie, Liv. I, §14, p. 205.
} 
leur disponibilité) entre les termes de la métaphore et en premier lieu son référent, l'imagination se trouve comme interdite et le discernement rendu impossible.

Une certaine critique moderniste ou postmoderne (on pense à Craig Owens et à Jean-François Lyotard") se retrouve ainsi (quoiqu'avec des degrés de conscience divers) confrontée à la même situation que ceux qui en ont décelé les indices dans l'art et la littérature d'après Auschwitz, que les œuvres qui en sont issues soient ou non clairement référencées à l'événement. Lorsque Luba Jurgenson écrit par exemple que, dans la littérature des camps, «l'image est présentée comme captée au moment même de son surgissement, n'ayant fait l'objet d'aucune élaboration $»^{10}$, soit exposée sur un mode antilittéraire, pour ne pas dire ici : antirhétorique, on devine non seulement que l'on peut retrouver des procédés comparables dans la littérature non concentrationnaire, mais combien l'hypotypose elle-même s'est altérée. S'agit-il d'ailleurs encore de cette figure lorsque l'image qu'elle met sous les yeux ne se présente pas comme un tableau, c'est-à-dire comme une instance de discernement, qu'elle n'emporte plus l'adhésion de ses lecteurs mais qu'elle les obsède longtemps après qu'ils l'ont eue sous les yeux, et que ce qu'elle donne à voir, enfin, semble inimaginable, incroyable et, en toute fin de compte, irregardable?

Quelque chose de cet ordre git pourtant dans le mot même d'hypotypose, auquel hupo, « sous » en grec, confère une valeur de latence, celle d'une « soustrace ». Tupos, en grec, c'est en effet l'empreinte - le «type » - et l'esquisse, mais ce ne saurait être l'image elle-même (eikôn), tout au plus son simulacre (eidôlon), voire son fantôme (phantasma). La mémoire, lit-on dans le Théétète, laisse une impression (tupos) « et ce qui a été imprimé, nous nous le rappelons et nous le savons, aussi longtemps que l'image [eidôlon] en est là »" ${ }^{11}$, c'est-à-dire en l'occurrence son double, dont la ressemblance (mimèsis) peut être troublante mais n'implique pas d'identité ${ }^{12}$. L'épicurisme distingua ensuite la « réplique conforme » (tupos) à son simulacre (eidolôn) de la réplique non conforme (phantasia) qui

\footnotetext{
${ }^{9}$ Owens a été l'un des premiers à commenter l'essai de Derrida et à y voir l'amorce d'un renouvellement de l'esthétique. Lyotard a quant à lui vu dans le vide laissé par le procès analogique lui-même tel que le décrit le $\$ 59$ la possibilité du sublime. $C f$., respectivement : C. Owens, « Détachement : à partir du parergon » [October, 1979], Proteus, $\mathrm{n}^{\circ}$ 2, septembre 2011, p. 44-49, en particulier p. 49; J.-F. Lyotard, Le Différend [1983], Paris, Minuit, 2007, en particulier p. 191-193.

${ }^{10}$ L. Jurgenson, L'Expérience concentrationnaire est-elle indicible?, Monaco, Rocher, 2003, p. 43.

${ }^{11}$ Platon, Théétète, trad. du grec ancien par M. Narcy, Paris, Garnier-Flammarion, 1994, 191c-e, p. 250.

${ }^{12}$ Jean-Pierre Vernant relève qu' eikôn est d'apparition plus tardive ( $\mathrm{V}^{\mathrm{e}}$ siècle avant J.-C.) qu'eidôlon, et contemporain de l'émergence du vocabulaire de la mimèsis appliqué aux figures plastiques ainsi que de l'avènement de la tragédie. Voir J.-P. Vernant, «Figuration et image », Mètis, vol. 5, $\mathrm{n}^{\mathrm{o}} 1-2,1990$, p. $225-238$.
} 
conduit au contraire à des jugements erronés parce que fantastiques ${ }^{13}$. Distinction qu'on ne trouve plus dans la rhétorique de Quintilien, lequel rapproche les phantasiai grecques des uisiones latines, "grâce auxquelles, écrit-il, les images des choses absentes sont représentées dans l'esprit, de telle sorte que nous croyons les discerner de nos yeux et les avoir présentes ${ }^{14}$. Le rhéteur confirme ainsi la crainte des philosophes et met entre les mains de ses successeurs classiques une définition de l'hypotypose que Barthes reconnaitra quant à lui comme celle du fantasme, précisément.

Bien que l'on imagine déjà combien la figure tire son pouvoir non seulement de sa puissance d'exposition (on hésite à écrire : d'exhibition) mais du trouble qu'elle introduit dans l'ordre du discernement, il importe de rappeler ce qu'elle fut avant Quintilien et après lui, avant d'acquérir cette signification très large dans la modernité. D'abord, comme y a insisté Jean-Pierre Aygon ${ }^{15}$, l'hypotypose ne fut conçue comme un «tableau » de type ekphrasis qu'à partir du II ${ }^{\mathrm{e}}$ siècle de notre ère, période à partir de laquelle elle peut être rapprochée de l'enargeia dont Quintilien avait déjà fait le plus bel ornement (ornatus) du discours $^{16}$. L'ekphrasis elle-même ne se définissait avant cette période que par sa puissance de visualisation obtenue avec force détails, et non par son objet; elle pouvait de surcroît être narrative. Selon Aygon, c'est à Lessing qu'il revient d'avoir durablement oblitéré cette dimension en liant dans son Laocoon (1766) «statisme » et description ekphrastique ${ }^{17}$.

Le siècle précédant celui de Lessing avait fait grand usage de l'hypotypose au point que l'on est tenté d'avancer qu'il en a redécouvert les pouvoirs ${ }^{18}$. C'est

${ }^{13}$ Sur ce point, $c f$. J.-F. Balaudé, Introduction à Épicure, Lettres, maximes, sentences, trad. du grec ancien par J.-F. Balaudé, Paris, Le Livre de Poche, 1994, p. 87.

${ }^{14}$ Cité in : Longin, Du sublime, trad. du latin par J. Pigeaud, Paris, Rivages Poche, 1993, p. 140, NdT.

${ }^{15}$ Et Michel Costantini à sa suite, ainsi que Ruth Webb plus récemment. $C f$. M. Costantini, «Écrire l'image, redit-on ", Littérature, $\mathrm{n}^{\circ}$ 100, 1995, p. 22-48; et R. Webb, Ekphrasis, Imagination, and Persuasion in Ancient Rhétorical Theory and Practice, Farnham, Ashgate, 2009.

${ }^{16}$ Notion qui se rapproche de celle d'œuvre (ergon) comme de parachèvement (parergon) au centre de laquelle se trouve l'enargeia aristotélicienne : «Il faut distinguer deux mots grecs employés par Aristote, prévient Colette Camelin : energeia, force en action (de ergon, l'action) par opposition à dunamis (force en puissance), et enargeia, vue claire et distincte, évidence (de argos, blanc, rapide, brillant). Est enarges (euidens), ce qui se rend visible avec force, ce qui frappe vivement les yeux et l'imagination dans une narration ou une description et dans une œuvre d'art plastique ou une performance spectaculaire ». C. Camelin, «Avant-Propos. L'intensité : forces, formes, variations », in : M. Briand, C. Camelin, L. Louvel (dir.), L'Intensité. Formes et forces, variations et régimes de valeur, Rennes, La Licorne, Presses Universitaires de Rennes, 2011, p. 7-38, ici p. 14.

${ }^{17}$ Sur ces points, $c f$. J.-P. Aygon, «L'ecphrasis et la notion de description dans la rhétorique antique », Pallas, $\mathrm{n}^{\circ}$ 41, 1994, p. 41-56, en particulier p. 46-47. Dans sa préface aux Tableaux (Eikones) de Philostrate, Pierre Hadot rappelle également que, pour ce dernier, « la parole peut peindre, aussi bien et mieux même que le trait du pinceau ». P. Hadot, préface à Philostrate, La Galerie de tableaux, trad. du grec ancien par A. Bougot, Paris, Les Belles Lettres, 2013, p. XIV.

${ }^{18} \mathrm{Par}$ « redécouverte », on entend la prise de conscience explicite desdits pouvoirs. Bien qu'elle fût évidemment connue de lui, Antoine Fouquelin n'inclut pas l'hypotypose dans sa Rhétorique 
en effet à cette période qu'elle semble déployer véritablement sa puissance symbolique en liant rhétorique et politique sous l'égide de la peinture. Ou pour le dire autrement, en termes de sémiostylistique cette fois : l'hypotypose s'affirme alors consciemment comme un "relais intersémiotique »" ${ }^{19}$, en ce qu'elle se situe à l'intersection de plusieurs régimes sémiotiques, ce dont la notion de figure est exemplaire, «car au XVII ${ }^{\mathrm{e}}$ siècle, écrit Lucile Gaudin, faire voir, c'est faire croire $»^{20}$. Par-là, non seulement « la rhétorique 'mord' sur le littéraire $»^{21}$, comme le relevait Barthes au moment de l'avènement du «morceau », mais elle déborde sur tout le champ de la représentation avec des velléités d'annexion; à ceci près que le trouble qu'elle instaure se retourne sur cela même qui le fait advenir dans la mesure où le discours tend vers cela qu'il ne peut atteindre : le tableau.

Louis Marin a par exemple montré qu'afin de faire croire objectivement (et donc universellement) à la réalité des faits qui y sont décrits, le projet d'historiographie royale que soumit Paul Pellisson à Louis XIV devait en passer par l'effacement des marqueurs de subjectivité, ce qui est un des traits saillants de l'hypotypose. «Personne ne parle ici, remarque Marin. Le roi se raconte lui-même dans son histoire. Étrange effet d'oubli du dire pour que le dit s'inscrive comme mémoire $»^{22}$. Le comble de cet effacement est atteint une première fois dans l'hypotypose narrative, en laquelle se transmet «la symbolique du Pouvoir $»^{23}$, et une seconde fois (mais elle lui est corrélative), dans le tableau d'histoire, qui est « l'ultime référence du récit historique futur et sa suprême justification ${ }^{24}:$ : Pourquoi ? demande Marin. Parce que seul, le tableau, par sa substance et les insurpassables contraintes de la représentation narrative iconique, donne nécessairement à voir le récit comme modèle a-chronique, au présent, dans l'espace limité de sa toile et dans l'ordre de coexistence de ses parties $»^{25}$.

françoyse de 1555, peut-être parce qu'elle lui paraît être une forme spontanée, comme le suggère Alex Gordon. De même, seul Jacques Peletier du Mans parmi les poètes de la Pléiade (mais il fut aussi grammairien) emploie le terme, Du Bellay lui préférant le terme d' " énergie ». Sur ces points, cf. Alex L. Gordon, "Les figures de rhétorique au XVI siècle », L'Information Grammaticale, $\mathrm{n}^{\circ}$ 75, 1997, p. 15-21, ainsi que H. Morier, Dictionnaire de poétique et de rhétorique, op. cit., "Hypotypose », p. 530. Reste que le principe même des discours en vers formés par Ronsard comme par d'Aubigné tend vers l'hypotypose, ce dernier introduisant même une dimension testimoniale dont on va voir la proximité avec la figure lorsqu'il écrit au début des Tragiques : «Car mes yeux sont tesmoins du sujet de mes vers ». A. d'Aubigné, Les Tragiques [1616], Paris, GarnierFlammarion, 1968, v. 371, p. 68.

${ }^{19}$ Sur cette qualité de l'hypotypose, cf. G. Molinié, Sémiostylistique. L'effet de l'art, Paris, Presses Universitaires de France, 1998, p. 42.

${ }^{20}$ L. Gaudin, «Du figural et du représentatif. Approche rhétorique et intersémiotique dans la littérature du XVII ${ }^{\mathrm{e}}$ siècle », L'Information Grammaticale, $\mathrm{n}^{\circ}$ 93, 2002, p. 48-49, ici p. 49.

${ }^{21} \mathrm{R}$. Barthes, L'Aventure sémiologique, op. cit., «L'ancienne rhétorique » [1965], p. 102.

${ }^{22}$ L. Marin, "Pouvoir du récit et récit du pouvoir », Actes de la recherche en sciences sociales, vol. 25 , janvier 1979 , p. 23-43, ici p. 39.

${ }^{23}$ Ibid., p. 40.

${ }^{24}$ Ibid., p. 42.

${ }^{25}$ Ibid. 
À l'inverse, on pourrait soutenir, toujours d'après Marin, que lorsque la peinture parvient à cette éloquence qui consiste à « faire voir» la voix qui lui manque, elle atteint «le comble de l'entreprise nommée représentation de peinture $»^{26}$, et réalise alors sa propre hypotypose. (Ne serait-ce pas d'ailleurs le comble de cette figure qu'en faisant voir ce qu'elle fait entendre elle obtienne de son auditoire le silence, ainsi que le chant y réussit parfois en ramenant toutes choses à sa présence ?) Mais si cette tentation précède le XVII siècle, au moins du côté des peintres $^{27}$, il est plus singulier que la forme hypotypique de la narration du pouvoir trouve en la critique d'art diderotienne ses prolongements les plus flagrants. Elle se compose en effet, note encore Marin, d' ' énoncés de 'faits' constatifs. Personne ne parle ici, poursuit-il. Les événements s'énoncent au fur et à mesure qu'ils se produisent à l'horizon de l'histoire $»^{28}$; d'où le recours au présent. C'est en cela que Diderot peut être qualifié par Marin de « descripteur fantaisiste » : en ce qu'il se crée des fantômes de tableaux qui hantent d'autant plus ceux qui les «lisent » qu'ils semblent émaner d'eux sans intermédiaire ${ }^{29}$.

À quelques années près, César Dumarsais aurait pu être de ces lecteurs, qui reprend peu ou prou dans son traité paru en 1730 la définition de Quintilien : "C'est lorsque, dans les descriptions, on peint les faits dont on parle, comme si ce qu'on dit était actuellement devant les yeux ; on montre, pour ainsi dire, ce qu'on ne fait que raconter; on donne en quelque sorte l'original pour la copie, les objets pour les tableaux $»^{30}$. Afin de justifier qu'il compte l'hypotypose dans sa tropologie, Dumarsais relève qu'elle a souvent pour amorce (amorce paradoxale en vérité, pour ne pas dire trompeuse, puisqu'elle se présente comme un embrayeur de mimèsis) un changement de temps, ce que l'auteur de L'Institution oratoire avait signalé et désigné sous le terme grec de metastasis : « Je ne mets ici cette figure au rang des Tropes, écrit Dumarsais, que parce qu'il y a quelque

${ }^{26}$ « Aux marges de la peinture : voir la voix», 1988, L. Marin, De la représentation, Paris, Gallimard, Seuil, 1994, p. 330.

${ }^{27}$ La tentative fut parfois plus littérale encore, ainsi que l'a relevé Victor Stoïchita, comme lorsque « le Titien fait tout son possible pour convaincre le spectateur érudit du Camerino d'Alabastro que l'ekphrasis [en l'occurrence de Philostrate] aurait pu se dérouler devant son propre tableau ». V. I. Stoïchita, Figures de la transgression, Genève, Droz, 2013, p. 30.

${ }^{28}$ L. Marin, Des pouvoirs de l'image. Gloses, Paris, Seuil, 1993, «Le descripteur fantaisiste », p. 87.

${ }^{29}$ Michael Fried écrit en ce sens que, pour Diderot, «l'unité d'action et plus encore celle de l'ensemble du tableau devaient produire l'illusion du dynamisme interne, du mouvement et de la force coercitive de la causalité elle-même ». M. Fried, La Place du spectateur. Esthétique et origines de la peinture moderne [1980], trad. de l'anglais par C. Brunet, Paris, Gallimard, 1990, p. 87.

${ }^{30}$ C. Dumarsais, Des tropes, ou des différents sens dans lesquels on peut prendre un même mot dans une même langue [1730], Paris, Périsse et Compère, 1811, p. 91. 
sorte de Trope à parler du passé comme s'il était présent $»^{31}$. Cette simple remarque fait plus que renforcer la dimension fantasmatique de l'hypotypose, elle l'ente sur l'axe du temps et l'ouvre au registre d'une mémoire à la fois vive et obscure parce que mal délimitée, une mémoire offusquée parce que pour partie du moins invraisemblable ; il est vrai que sa présence même est anormale. Lointainement peut-être, Bernard Dupriez prend acte de cette ouverture lorsqu'il note la parenté de l'hypotypose avec l'hallucination comme avec l'évocation, à la fois « de pure forme » et « au sens fort », comprenant « quelque chose de surréel ou d'obsessionnel ». « Il y a aussi évocation, ajoute Dupriez, quand on revit un événement passé $»^{32}$.

Mais c'est sur un traité du XVII ${ }^{\mathrm{e}}$ siècle, La Rhétorique ou l'art de parler, publié en 1675 par Bernard Lamy, que Roland Barthes fait fond afin de mettre en évidence la fantasmatique à l'œuvre dans le théâtre de Racine. Lamy écrit en effet que les hypotyposes «forment une image qui tient lieu des choses mêmes » ${ }^{33}$, ce qui est la meilleure définition du fantasme selon l'auteur du Sur Racine ${ }^{34}$ là où celui de La Rhétorique l'apparente à « une espece d'enthousiasme qui fait qu'on s'imagine voir ce qui n'est point présent $»^{35}$. S'il en est ainsi pour Barthes, c'est que, selon lui, chez Racine, «l'acte est l'impureté même » ${ }^{36}$, et l'image la promesse d'un arrêt sur image, par quoi ses corps tragiques accèdent à une forme d'érotisme de la souvenance : «les moments réussis de l'érotique racinienne, écrit Barthes, sont toujours des souvenirs $»^{37}$. À son climax, l'hypotypose est réitérée : "Ainsi le tableau racinien est toujours une véritable anamnèse, insiste Barthes : le héros tente sans cesse de remonter à la source de son échec; mais comme cette source est son plaisir même, il se fige dans son passé : Éros est en lui une force rétrospective : l'image est répétée, jamais dépassée $»^{38}$.

Aux yeux de Barthes, cette fantaisie de l'hypotypose s'est dissoute dans le réalisme littéraire qui, tout à son référent réel, évite à « la description réaliste [...] de se laisser entraîner dans une activité fantasmatique (précaution que l'on croyait nécessaire à 1' 'objectivité' de la relation) ${ }^{39}$; c'est l'avènement du neutre, du constatif, écrit encore Barthes. En réalité, l'hypotypose n'a pas complètement disparu, elle a seulement perdu de son éclat, elle est entrée, d'une certaine manière,

${ }^{31}$ Ibid., p. 93. À la fois par rigidité classificatrice et parce que, comme pour Fouquelin, il s'agit d'une figure commune à tous les littérateurs, Pierre Fontanier ne la retient pas quant à lui comme trope. Voir P. Fontanier, Les Figures du discours [1827-1830], Paris, Garnier-Flammarion, 1977, p. 266 et 390.

${ }_{32}$ B. Dupriez, Gradus. Les procédés littéraires (Dictionnaire) [1984], Paris, UGE, 1995, p. 345.

${ }^{33}$ B. Lamy, La Rhétorique ou l'art de parler [1675], La Haye, Pierre Paupie, 1737, p. 153.

${ }^{34}$ Cf. R. Barthes, Sur Racine [1963], Paris, Seuil, 1979, p. 23.

${ }^{35}$ B. Lamy, La Rhétorique ou l'art de parler, op. cit., p. 153.

${ }^{36}$ R. Barthes, Sur Racine, op. cit., p. 13.

${ }^{37}$ Ibid., p. 22.

${ }^{38}$ Ibid., p. 28.

${ }^{39}$ R. Barthes, «L'effet de réel », Communications, 1968, in : G. Genette, T. Todorov (dir.), Littérature et réalité, Paris, Seuil, 1982, p. 86. 
en décrépitude ; elle a cessé de se poser elle-même en figure du discernement pour apparaître comme une image persistante, de manière presque neutre, constative, comme à bas bruit, mais d'autant plus durable qu'elle semble là aussi objective. Lorsque Barthes écrit que, dans le réalisme, «le 'réel' est réputé se suffire à luimême, qu'il est assez fort pour démentir toute idée de 'fonction', que son énonciation n'a nul besoin d'être intégrée dans une structure et que l'avoir-été-là des choses est un principe suffisant de sa parole $»^{40}$, il rejoint d'assez près l'analyse que fait Marin du récit de pouvoir que l'on a mentionnée, à cette différence près que le réel semble avoir supplanté le roi et son biographe, et son eidôlon leurs effigies, et que le vertige de détail risque en effet de nuire au relief de la scène ainsi décrite ${ }^{41}$.

Barthes retrouva plus tard dans la photographie cette «force constative » que l'on peut apparenter à l'énergie de l'hypotypose, d'autant plus qu'il précise qu'en ce cas précis elle ne porte pas « sur l'objet, mais sur le temps » ${ }^{42}$, manière d'introduire ici l'idée que tout un pan de l'art contemporain, et non seulement de la littérature, répond à ce régime hypotypique de basse intensité où la description remplace la narration, l'initiale le nom, et le fond la figure, au risque de ne produire à la vue que des faces biffées d'après lesquelles toutes tentatives de reconnaissance et de discernement sont dès l'abord compromises. Sous ce rapport, l'hypotypose contemporaine - et dans une certaine mesure c'est aussi le cas de la photographie - est une déposition, à la fois au sens d'une destitution de son aura rhétorique passée et à celui d'un témoignage de ce qui a eu lieu, même s'il paraît invraisemblable à ceux qui le reçoivent. Bien que le témoignage dépasse toujours le cadre de la déposition de type judiciaire, celle-ci en reste le parangon, comme l'a relevé Renaud Dulong ${ }^{43}$, et son éthique détermine pour partie son esthétique ${ }^{44}$ : elle l'imprègne et l'oriente, et c'est en ce sens que l'hypotypose a à voir avec le testimonial, bien que sa capacité à altérer la distinction entre les temps de l'énonciation et de l'énoncé suffise à la distinguer du témoignage à strictement parler.

Il revient à Yves Le Bozec d'avoir montré ce trait singulier selon lequel, dans l'hypotypose, la « mise de côté du narrateur n'est pas synonyme de la disparition du locuteur », dans la mesure, écrit-il, où, d'une part, « il peut rester là

\footnotetext{
${ }^{40}$ Ibid., p. 87.

${ }^{41}$ Morier, qui estime que l'hypotypose a pactisé avec le réalisme, observe au contraire dans le nouveau roman (en l'occurrence La Modification de Michel Butor) une atténuation de l'hypotypose, «parce que les détails, écrit-il, s'y trouvent d'égale valeur et noyés dans un océan d'autres détails ». H. Morier, Dictionnaire de poétique et de rhétorique, op. cit., "Hypotypose », p. 531.

${ }^{42}$ R. Barthes, La Chambre claire. Note sur la photographie, Paris, Cahiers du Cinéma, Gallimard, Seuil, 1980, p. 138-139.

${ }^{43}$ Cf. R. Dulong, Le Témoin oculaire. Les conditions sociales de l'attestation personnelle, Paris, École des Hautes Études en Sciences Sociales, 1998, p. 41.

${ }^{44} C f$. sur ce point C. Lacoste, "Approche sémantique d'un genre littéraire : le témoignage », in : D. Ablali, S. Badir, D. Ducard (dir.), Documents, textes, euvres. Perspectives sémiotiques, Rennes, Presses universitaires de Rennes, 2014, p. 179-192, ici p. 186.
} 
comme témoin, tant qu'il n'intervient pas dans la figure », et que, d'autre part, «sa présence passive accroît l'effet de sidération, en même temps qu'elle accrédite la scène ${ }^{45}$. Le corps réduit à sa faculté énonciatrice demeure cependant essentiel en tant que présence d'attestation des événements passés, voire comme preuve corporelle, phénomène constitutif de toute théorie du témoignage : «Le corps du témoin présentifie l'événement, écrit en ce sens Dulong, en établissant une continuité physique entre ce passé et le présent de la rencontre ${ }^{46}$. Mais, dans le cadre de l'hypotypose, le critère de non interférence du témoin en tant que personne avec la configuration de son témoignage définit pour lui une position de détachement, de retrait, voire, suggère Le Bozec, de dissimulation. Dans l'hypotypose extraite d'Andromaque qu'il examine, le héros éponyme, écrit Le Bozec, « devient personnage à la personne trois, se dédoublant du narrateur afin de n'être plus qu'un élément du tableau: contrairement à une peinture romantique qui placerait le poète assis seul sur un rocher au centre de la toile, ajoute Le Bozec, l'hypotypose le cache au mieux parmi la foule $»{ }^{47}$.

Une dernière conséquence abonde dans le sens du détachement caractéristique de la déposition : « la première conséquence de l'abolition du point de vue, écrit Le Bozec, est l'exclusion du lyrisme : l'hypotypose s'interdit toute expression des sentiments du locuteur $\rangle^{48}$, par quoi elle s'écarte encore un peu plus, insiste-t-il, de la description romantique. Comme figure, l'hypotypose provoque un effet en partie neutralisant sur les autres figures, a fortiori lorsqu'elle rapporte une mort ou un drame, l'hypotypose pouvant en l'occurrence exclure du cadre qu'elle institue les métaphores, comme si, déjà là, le réel, fût-il un phantasme, obligeait en quelque sorte la fiction à n'opérer d'autre déplacement de sens que celui de la mémoire. Car c'est la vivacité du souvenir qui détermine en quelque manière celle de son porteur, toute l'intensité de l'hypotypose narrée reposant sur le sens et le ton qu'il donne à ses paroles (sur leur teneur), et non sur son imagination puisqu'elle est nécessairement bridée par la factualité du passé qu'il rapporte et, ce faisant, qu'il expose sous les yeux des auditeurs - en le leur dépeignant. Comme le suggère Le Bozec, l'auteur d'une hypotypose peut moins encore être narrateur au sens plein du terme, dans la mesure où il est absent au récit, non pas en tant que tel mais en tant qu'il en est l'inventeur; son auctoritas lui vient de son vécu, non de sa fantaisie.

\footnotetext{
${ }^{45}$ Y. Le Bozec, «L'hypotypose : un essai de définition formelle », L'Information Grammaticale, $\mathrm{n}^{\mathrm{o}}$ 92, 2002, p. 3-7, ici p. 5.

${ }^{46}$ R. Dulong, Le Témoin oculaire. Les conditions sociales de l'attestation personnelle, op. cit., p. 192. Sur cette puissance de "présentification » du témoignage comme « dimension figurative», cf. J.-P. Pierron, «La dimension figurative du témoignage : de la poétique à l'éthique », in: E. Alloa, S. Kristensen (dir.), Témoignage et survivance, Genève, MétisPresses, 2014, p. 239-258 ; sur l'hypotypose, $c f$. en particulier p. 247-248.

${ }^{47}$ Y. Le Bozec, «L'hypotypose : un essai de définition formelle », op. cit., p. 5.

${ }^{48}$ Ibid.
} 
En d'autres termes, ce qu'il raconte, il l'a vu, et son récit ne parait fantastique que parce qu'il le hante et qu'il le voit encore, qu'il en porte en lui une image, ayant sa contenance propre, sans quoi il ne pourrait le dire au présent. Il y a ainsi une sujétion du témoin à son expérience comme il y a une obligation du témoignage envers elle, et cette relation définit un véritable espace d'allocution et de réception. L'hypotypose est en effet adressée, car, si elle suppose l'effacement du locuteur, elle présume l'existence d'un allocuteur, auquel sa teneur échappe en partie cependant, soit qu'elle subjugue, soit qu'elle horrifie son destinataire, comme elle se détache de celui qui la porte ; l'hypotypose est toujours plus ou moins une dérive où les mots se succèdent sans personne pour les retenir. Et s'il est évidemment regrettable que l'absolutisation de la figure vers laquelle tend Le Bozec le conduise à référer finalement l'hypotypose à une position énonciatrice du divin, issue qui structure aussi son analyse de deux moments quasihypotypiques d'Athalie ${ }^{49}$, cette solution indique négativement qu'un vide se tient aujourd'hui au creux de cette figure que tout « essai de définition formelle » ne peut manquer d'apercevoir, et risque donc de chercher à combler. Le divin serait ici l'image manquante, celle à même d'enclencher le processus analogique faisant défaut à la figure revenue à elle seule.

Que faire en effet d'une figure qui « tourne à vide » philosophiquement, et n'a plus même l'ornement pour finalité intermédiaire, sinon constater qu'elle structure malgré cela l'imaginaire contemporain sur le mode du brouillage des temporalités, et signe l'immixtion non seulement du passé dans le présent, mais de l'image d'avant dans l'appréhension du présent d'après ?

C'est ce qu'avait observé Gilbert Durand lorsqu'il avançait que «le style de l'hypotypose » provoque « la répétition des images, et par là même la réversibilité du temps, [qu'il] anéantit le concept même de temps $»^{50}$. C'est le sens aussi qu'a donné François Hartog à la notion de présentisme, où « le présent nouveau se reconnaît endetté $»^{51}$. C'est, enfin, à la maîtrise de cette dette qu'introduit l'hypotypose que cherchait à répondre Paul Ricœur dans La Mémoire, l'histoire, l'oubli ; toute l'entreprise de l'ouvrage, depuis sa note d'orientation liminaire jusqu'à ses conclusions, visant à découpler l'imagination de la mémoire afin de prévenir « la menace permanente de confusion entre remémoration et imagination, résultant de

\footnotetext{
${ }^{49} C f$. Y. Le Bozec, «Les frontières de l'hypotypose : Le songe d'Athalie et la prophétie de Joad », L'Information Grammaticale, $\mathrm{n}^{\circ} 100,2004$, p. 26-30, ici p. 30.

${ }^{50}$ G. Durand, Les Structures anthropologiques de l'imaginaire [1969], Paris, Bordas, 1981, p. 408.

${ }^{51}$ F. Hartog, Régimes d'historicité. Présentisme et expériences du temps [2003], Paris, Seuil, 2012, p. 232. On a bien affaire à une nouvelle forme d'hypotypose, distincte de celles que l'on trouve en particulier chez les historiens du XIX ${ }^{\mathrm{e}}$ siècle dont Michelet serait le représentant le plus éminent. Sur ce point, voir P. Petitier, "Entre concept et hypotypose : l'histoire au XIX siècle », Romantisme, $\mathrm{n}^{\mathrm{o}} 2,2009$, p. 69-80.
} 
ce devenir-image du souvenir $»^{52}$, c'est-à-dire, en toute fin de compte, à empêcher que les fantômes n'investissent l'écriture et les représentations l'histoire. «La hantise est à la mémoire collective ce que l'hallucination est à la mémoire privée, écrit Ricœur, une modalité pathologique de l'incrustation du passé au cœur du présent, laquelle fait pendant à l'innocente mémoire-habitude qui, elle aussi, habite le présent, mais pour 'l'agir', dit Bergson, non point pour le hanter, c'est-à-dire le tourmenter $»^{53}$. Peut-être est-ce ce qui explique que Ricœur préfère à l'hypotypose le néologisme forgé d'après Gadamer de représentance ${ }^{54}$, alors même qu'il en partage toutes les qualités d'exposition. Dans tous les cas, il s'agit de retrouver les possibilités d'un ergon, d'un "œuvrer », qui désigne aussi pour Aristote le devoir de mener une vie achevée et active ${ }^{55}$. Une telle approche fait cependant l'économie de ce dont portent témoignage les hypotyposes actuelles, et, en détachant la mémoire dont elles sont porteuses de leur support, du parergon qu'est aussi l'hypotypose, elle rétablit dans ses droits une rhétorique et une esthétique qui ne laisse évidemment pas d'agir, mais dont le réel a cependant altéré le sens et sans doute définitivement rompu le cours.

\section{Bibliographie}

Aristote, Poétique, trad. du grec ancien par Jean Hardy, Paris, Gallimard, 2005

Aubigné, Agrippa d', Les Tragiques [1616], Paris, Garnier-Flammarion, 1968

Barthes, Roland, Sur Racine [1963], Paris, Seuil, 1979

Barthes, Roland, L'Aventure sémiologique [1985], Paris, Seuil, 1991, «L'ancienne rhétorique » [1965]

Barthes, Roland, «L'effet de réel », Communications, 1968, in: G. Genette, T. Todorov (dir.), Littérature et réalité, Paris, Seuil, 1982

Barthes, Roland, La Chambre claire. Note sur la photographie, Paris, Cahiers du Cinéma, Gallimard, Seuil, 1980

Camelin, Colette, «Avant-Propos. L'intensité : forces, formes, variations », in: Michel Briand, Colette Camelin, Liliane Louvel (dir.), L'Intensité. Formes et forces, variations et régimes de valeur, Rennes, La Licorne, Presses Universitaires de Rennes, 2011, p. 7-38

Costantini, Michel, «Écrire l'image, redit-on », Littérature, $n^{\circ}$ 100, 1995, p. 22-48

Derrida, Jacques, La Vérité en peinture [1978], Paris, Flammarion, 1996

Dulong, Renaud, Le Témoin oculaire. Les conditions sociales de l'attestation personnelle, Paris, École des Hautes Études en Sciences Sociales, 1998

Dumarsais, César, Des tropes, ou des différents sens dans lesquels on peut prendre un même mot dans une même langue [1730], Paris, Périsse et Compère, 1811

Dupriez, Bernard, Gradus. Les procédés littéraires (Dictionnaire) [1984], Paris, UGE, 1995

Durand, Gilbert, Les Structures anthropologiques de l'imaginaire [1969], Paris, Bordas, 1981

Épicure, Lettres, maximes, sentences, trad. du grec ancien par Jean-François Balaudé, Paris, Le Livre de Poche, 1994

Fontanier, Pierre, Les Figures du discours [1827-1830], Paris, Garnier-Flammarion, 1977

${ }^{52}$ P. Ricœur, La Mémoire, l'histoire, l'oubli [2000], Paris, Seuil, coll. « Points essais », 2003, p. 7.

${ }^{53}$ Ibid., p. 65.

${ }^{54}$ Cf. Ibid., p. 367, note.

${ }^{55}$ Cf. P. Ricour, Parcours de la reconnaissance. Trois études [2004], Paris, Gallimard, 2005, p. 137. 
Fried, Michael, La Place du spectateur. Esthétique et origines de la peinture moderne [1980], trad. de l'anglais par Claire Brunet, Paris, Gallimard, 1990

Gaudin, Lucile, «Du figural et du représentatif. Approche rhétorique et intersémiotique dans la littérature du XVII ${ }^{\mathrm{e}}$ siècle », L'Information Grammaticale, ${ }^{\circ}$ 93, 2002, p. 48-49

Gordon, Alex L., "Les figures de rhétorique au XVI ${ }^{\mathrm{e}}$ siècle », L'Information Grammaticale, $\mathrm{n}^{\mathrm{o}} 75,1997$, p. $15-21$

Hartog, François, Régimes d'historicité. Présentisme et expériences du temps [2003], Paris, Seuil, 2012

Jurgenson, Luba, L'Expérience concentrationnaire est-elle indicible ?, Monaco, Rocher, 2003

Kant, Emmanuel, Critique de la faculté de juger [1790], trad. de l'allemand par A. Renaut, Paris, Garnier-Flammarion, 1995

Lacoste, Charlotte, "Approche sémantique d'un genre littéraire : le témoignage », in: Driss Ablali, Sémir Badir, Dominique Ducard (dir.), Documents, textes, euvres. Perspectives sémiotiques, Rennes, Presses universitaires de Rennes, 2014, p. 179-192

Lamy, Bernard, La Rhétorique ou l'art de parler [1675], La Haye, Pierre Paupie, 1737

Le Bozec, Yves, «L'hypotypose : un essai de définition formelle », L'Information Grammaticale, $n^{\circ}$ 92, 2002, p. 3-7

Le Bozec, Yves, «Les frontières de l'hypotypose : Le songe d'Athalie et la prophétie de Joad», L'Information Grammaticale, $\mathrm{n}^{\circ} 100,2004$, p. 26-30

Longin, Du sublime, trad. du latin par Jackie Pigeaud, Paris, Rivages Poche, 1993

Lyotard, Jean-François, Le Différend [1983], Paris, Minuit, 2007

Marin, Louis, «Pouvoir du récit et récit du pouvoir », Actes de la recherche en sciences sociales, vol. 25, janvier 1979, p. 23-43

Marin, Louis, Des pouvoirs de l'image. Gloses, Paris, Seuil, 1993, «Le descripteur fantaisiste »

Marin, Louis, De la représentation, Paris, Gallimard, Seuil, 1994, « Aux marges de la peinture : voir la voix » [1988]

Mesnard, Philippe, Témoignage en résistance, Paris, Stock, 2007

Molinié, Georges, « Problématique de la répétition », Langue française, $n^{\circ} 101,1994$, p. 102-111

Molinié, Georges, Sémiostylistique. L'effet de l'art, Paris, Presses Universitaires de France, 1998

Morier, Henri, Dictionnaire de poétique et de rhétorique [1961], Paris, Presses Universitaires de France, 1989

Owens, Craig, « Détachement : à partir du parergon » [October, 1979], Proteus, $\mathrm{n}^{\circ}$ 2, septembre 2011, p. 44-49

Petitier, Paule, «Entre concept et hypotypose : l'histoire au XIX ${ }^{\mathrm{e}}$ siècle », Romantisme, $\mathrm{n}^{\circ}$ 2, 2009, p. $69-80$

Pierron, Jean-Philippe, «La dimension figurative du témoignage : de la poétique à l'éthique », in : Emmanuel Alloa, Stefan Kristensen (dir.), Témoignage et survivance, Genève, MétisPresses, 2014, p. 239-258

Philostrate, La Galerie de tableaux, trad. du grec ancien par Auguste Bougot, Paris, Les Belles Lettres, 2013

Platon, Théétète, trad. du grec ancien par Michel Narcy, Paris, Garnier-Flammarion, 1994

Ricœur, Paul, La Métaphore vive [1975], Paris, Seuil, 1997

Ricœur, Paul, Parcours de la reconnaissance. Trois études [2004], Paris, Gallimard, 2005

Ricœur, Paul, La Mémoire, l'histoire, l'oubli [2000], Paris, Seuil, coll. « Points essais », 2003

Stoïchita, Victor I., Figures de la transgression, Genève, Droz, 2013

Vernant, Jean-Pierre, « Figuration et image », Mètis, vol. 5, nº 1-2, 1990, p. 225-238

Webb, Ruth, Ekphrasis, Imagination, and Persuasion in Ancient Rhétorical Theory and Practice, Farnham, Ashgate, 2009 


\section{Paul Bernard-Nouraud}

Associé au Centre de Recherche sur les Arts et le Langage de l'École des Hautes Études en Sciences Sociales de Paris, Paul Bernard-Nouraud est docteur en histoire et théorie de l'art. Il travaille sur les formes de la représentation et des imaginaires dans l'art d'après-Auschwitz. Il a notamment publié Figurer l'autre. Essai sur la figure du "musulman » dans les camps nazis (Paris, Kimé, 2013), et co-dirigé avec Luba Jurgenson Témoigner par l'image (Paris, Pétra, 2015), ainsi qu'un article sur le rôle des hypotyposes dans l'œuvre de Charlotte Delbo, in: Christiane Page (dir.), Charlotte Delbo. Engagement, univers concentrationnaire, æuvre, Rennes, PUR, 2014. 